\title{
Modified Chebyshev collocation method for delayed predator-prey system
}

\section{J. Dengata ${ }^{1}$ and Shufang $\mathrm{Ma}^{1 *}$}

\section{"Correspondence:}

shufangma@nefu.edu.cn; dingatal@yahoo.com

'Department of Mathematics, Northeast Forest University, Harbin, China

\begin{abstract}
In this study, the approximate solutions of the predator-prey system with delay have been obtained by using the modified Chebyshev collocation method. The main technique is that this method transforms the original problem into a system of nonlinear algebraic equations. By using the residual function of the operator equations, error differential equations are constructed and thus the approximate solutions are corrected. A numerical example is given to confirm the reliability and applicability of the method, and comparisons with existing results are given. The numerical results show that the obtained solutions are in good agreement with earlier studies.
\end{abstract}

Keywords: Predator-prey system; Delay; Modified Chebyshev polynomial; Collocation method; Residual correction

\section{Introduction}

The predator-prey model is the essential model in studying the population dynamics of many species, which was initially proposed by Lotka and Volterra (see $[1,2])$. It has wide application in various research areas, such as chemical processes (see [3, 4]), bioparticles granulation (see [5]), the interaction of microorganisms and ecosystems (see [6, 7]). In recent years, many researchers have worked on the Lotka-Volterra type predator-prey system. In particular, Zhu et al. [8] focused on competitive Lotka-Volterra model in random environments. Li et al. [7] studied the canard phenomenon for predator-prey systems with response functions of Holling types. Badri et al. [9] dealt with the stabilization of the feasible equilibrium point of a special class of nonlinear quadratic systems known as Lotka-Volterra systems.

In order to get a more realistic model, the time delay has been taken into account in the predator-prey system. The analytical and dynamical aspects of such time delay models have been studied extensively by many researchers (see [10-12]). Also, there have been many studies interested in obtaining numerical solutions to the predator-prey system. For example, Capobianco [13] solved the numerical solution of Lotka-Volterra by using high performance parallel numerical methods. Susmita Paul [14] explained how to solve the Lotka-Volterra predator-prey model by using the Runge-Kutta-Fehlberg (RKF)

(c) The Author(s) 2020. This article is licensed under a Creative Commons Attribution 4.0 International License, which permits use, sharing, adaptation, distribution and reproduction in any medium or format, as long as you give appropriate credit to the original author(s) and the source, provide a link to the Creative Commons licence, and indicate if changes were made. The images or other third party material in this article are included in the article's Creative Commons licence, unless indicated otherwise in a credit line to the material. If material is not included in the article's Creative Commons licence and your intended use is not permitted by statutory regulation or exceeds the permitted use, you will need to obtain permission directly from the copyright holder. To view a copy of this licence, visit http://creativecommons.org/licenses/by/4.0/. 
method. Gokmen [15] used Taylor's collocation method to find the numerical solution of the predator-prey system with delay.

Nowadays, more and more attention is focused on the fractional predator-prey dynamical system. However, few of the fractional equations can be solved explicitly, but the broad application attracts many authors to devote themselves to numerical methods of these equations (see [16-30]). Very recently, many numerical methods have been developed for solving the fractional predator-prey dynamical system. Particularly, hybrid analytic approach [31], Haar wavelet and Adams-Bashforth-Moulton methods [32], Bernstein wavelet and Euler methods [33], and a numerical scheme based on the homotopy analysis transform technique [34].

Chebyshev polynomials have become very important in numerical analysis. They are widely used because of their advantages, such as the roots of the first kind of Chebyshev polynomials (Gauss-Lobatto nodes) being used in polynomial interpolation for minimizing the Runge phenomena, providing the best uniform approximation of polynomials in continuous functions (see [35-37]). Most commonly used techniques with Chebyshev polynomials have been examined in [38-40] and the references therein.

Motivated by the above discussion, we are mainly interested in applying a modified Chebyshev collocation method for the time-delay predator-prey model in [10] as follows:

$$
\left\{\begin{array}{l}
y_{1}^{\prime}(t)=y_{1}(t)\left[r_{1}-a_{11} y_{1}(t-\tau)-a_{12} y_{2}(t)\right], \\
y_{2}^{\prime}(t)=y_{2}(t)\left[-r_{2}+a_{21} y_{1}(t)-a_{22} y_{2}(t)\right],
\end{array} \quad t \in[0, T]\right.
$$

with initial conditions

$$
\begin{aligned}
& y_{1}(0)=\alpha, \\
& y_{2}(0)=\beta,
\end{aligned}
$$

where $y_{1}(t)$ and $y_{2}(t)$ are interpreted as the densities of prey and predator respectively, $r_{1}>0$ is the growth rate of prey in the absence of predators, $a_{11}>0$ denotes the selfregulation constant of prey, $a_{12}>0$ describes the predation of prey by predators, $r_{2}>0$ is the death rate of predators in the absence of prey, $a_{21}>0$ is the conversion rate for predators, $a_{22}>0$ describes the intraspecific competition among predators and $\tau$ is the generation time of the prey species, $\alpha, \beta$ are constant.

The objective of this paper is to obtain the approximation solutions of system (1) in the form of truncated Chebyshev series. The primary benefit of this method is the nonlinear term that can easily be dealt with without any extra efforts. Other advantages include this method being nondifferential, nonintegral, and easily implemented on a computer.

This paper is organized as follows: In Sect. 2, a brief review of the shifted Chebyshev polynomial and its properties is provided. In Sect. 3, we apply the collocation method for system (1) using the shifted Chebyshev polynomial. In Sect. 4, we construct a fundamental matrix equation for system (1). In Sect. 5, we introduce the technique of residual error correction in order to check the accuracy of the method. Finally, a numerical example is presented to verify the efficiency and accuracy of the proposed method. 


\section{Shifted Chebyshev polynomials and their properties}

In this section, we introduce Chebyshev polynomials. The Chebyshev polynomials are the sets of orthogonal polynomials and they are simply related to the trigonometric functions (see $[41,42]$ ) by the formula

$$
T_{n}(\cos \theta)=\cos (n \theta)
$$

with $\theta \in[0, \pi]$. The Chebyshev polynomial $T_{n}(x)$ of the first kind is a polynomial in $x$ of degree $n$, defined by the following relation [43]:

$$
T_{n}(x)=\cos (n \arccos (x)), \quad n=0,1, \ldots, x \in[-1,1] .
$$

Since we use polynomial on $t \in[0, L]$ for any real number $L>0$, we can obtain the shifted Chebyshev polynomials $T_{n}^{*}(t)=T_{n}\left(\frac{2 t}{L}-1\right)$ by introducing the change of variable $x=2 t / L-$ $1, t \in[0, L]$. The shifted Chebyshev polynomial $T_{n}^{*}(t)$ satisfies the recurrence relation as follows:

$$
T_{(n+1)}^{*}(t)=2\left(\frac{2 t}{L}-1\right) T_{n}^{*}(t)-T_{(n-1)}^{*}(t), \quad n \in N
$$

with the boundary condition

$$
T_{n}^{*}(0)=(-1)^{n}, \quad T_{n}^{*}(L)=1 .
$$

And $T_{n}^{*}(t)$ satisfies the discrete orthogonality condition

$$
\sum_{k=0}^{N}{ }^{\prime \prime} T_{i}^{*}\left(t_{k}\right) T_{j}^{*}\left(t_{k}\right)= \begin{cases}0, & i \neq j, \\ N, & i=j=0, \\ \frac{N}{2}, & i=j \neq 0,\end{cases}
$$

where the interpolation points $t_{k}$ are chosen to be the Chebyshev-Gauss-Lobatto associated with the interval $[0, L]$ and $t_{k}=\frac{L}{2}\left(1-\cos \left(k \frac{\pi}{N}\right)\right), k=0,1,2, \ldots, N$. The summation symbol with double primes denotes a sum with both the first and last term halved [43].

\section{Method of solution}

Continuous and bounded functions $y_{s}(t)(s=1,2)$ can be approximated in terms of shifted Chebyshev polynomials in the interval $[0, L]$ as follows:

$$
y_{s N}(t)=\sum_{k=0}^{N}{ }^{\prime \prime} c_{s k} T_{k}^{*}(t) .
$$

Using the discrete orthogonality relation (3), coefficient $c_{s k}$ in (4) is given by

$$
c_{s k}=\frac{2}{N} \sum_{i=0}^{N}{ }^{\prime \prime} y_{s}\left(t_{i}\right) T_{k}^{*}\left(t_{i}\right) .
$$


Our aim is to obtain the unknown coefficients $y_{s}\left(t_{i}\right)$ for $i=0,1,2, \ldots, N$, and the method of solution being considered should be programmable in a computer.

From equations (4) and (5) we can obtain the function $y_{s N}(t)$ as follows:

$$
y_{s N}(t)=T(t) \cdot P \cdot Y_{s}
$$

where

$$
\begin{aligned}
& T(t)=\left[T_{0}^{*}(t), T_{1}^{*}(t), T_{2}^{*}(t), \ldots, T_{N}^{*}(t)\right], \\
& P=\left[\begin{array}{ccccc}
\frac{1}{2 N} T_{0}^{*}\left(t_{0}\right) & \frac{2}{2 N} T_{0}^{*}\left(t_{1}\right) & \ldots & \frac{2}{2 N} T_{0}^{*}\left(t_{N-1}\right) & \frac{1}{2 N} T_{0}^{*}\left(t_{N}\right) \\
\frac{1}{2 N} T_{1}^{*}\left(t_{0}\right) & \frac{2}{2 N} T_{1}^{*}\left(t_{1}\right) & \ldots & \frac{2}{2 N} T_{1}^{*}\left(t_{N-1}\right) & \frac{1}{2 N} T_{1}^{*}\left(t_{N}\right) \\
\vdots & \vdots & & & \vdots \\
\frac{1}{2 N} T_{N}^{*}\left(t_{0}\right) & \frac{2}{2 N} T_{N}^{*}\left(t_{1}\right) & \ldots & \frac{2}{2 N} T_{0}^{*}\left(t_{N-1}\right) & \frac{1}{2 N} T_{N}^{*}\left(t_{N}\right)
\end{array}\right], \\
& Y_{s}=\left[\begin{array}{llll}
\left.y_{s}\left(t_{0}\right), y_{s}\left(t_{1}\right), \ldots y_{s}\left(t_{N}\right)\right]^{\prime} .
\end{array}\right.
\end{aligned}
$$

We know that

$$
T^{\prime}(t)=T(t) \cdot K
$$

where $K=\frac{2}{L} M$ and $M$ is the operational matrix.

$$
M=\left[\begin{array}{cccccccc}
0 & 1 & 0 & 2 & 0 & 5 & \cdots & m 1 \\
0 & 0 & 4 & 0 & 8 & 0 & \cdots & m 2 \\
0 & 0 & 0 & 6 & 0 & 10 & \cdots & m 3 \\
\vdots & \vdots & \vdots & \vdots & \vdots & \vdots & \vdots & \vdots \\
0 & 0 & 0 & 0 & 0 & 0 & \cdots & 2 N \\
0 & 0 & 0 & 0 & 0 & 0 & \cdots & 0
\end{array}\right]_{(N+1) \times(N+1)}
$$

where $m 1, m 2$, and $m 3$ are respectively $N, 0,2 N$ for odd $N$ and $0,2 N, 0$ for even $N$. Then from the above equation we can write $y_{s N}^{\prime}(t)$ as follows:

$$
y_{s N}^{\prime}(t)=T(t) \cdot K \cdot P \cdot Y_{s}
$$

\section{Fundamental matrix equation for system (1)}

To obtain the fundamental matrix equations of system (1), we substitute equations (6) and (7) into system (1). We get the fundamental matrix system

$$
\left\{\begin{array}{l}
T(t) K P Y_{1}=T(t) P Y_{1}\left[r_{1}-a_{11} T(t-\tau) P Y_{1}-a_{12} T(t) P Y_{2}\right] \\
T(t) K P Y_{2}=T(t) P Y_{2}\left[-r_{2}+a_{21} T(t) P Y_{1}-a_{12} T(t) P Y_{2}\right]
\end{array}\right.
$$

Let

$$
P Y_{1}=Z_{1}, \quad P Y_{2}=Z_{2}
$$


We write equations (8) as follows:

$$
\begin{aligned}
& T(t) K Z_{1}-r_{1} T(t) Z_{1}+a_{11} T(t-\tau) Z_{1} T(t) Z_{1}+a_{12} T(t) Z_{2} T(t) Z_{1}=0 \\
& T(t) K Z_{2}+r_{2} T(t) Z_{2}-a_{21} T(t) Z_{1} T(t) Z_{2}+a_{22} T(t) Z_{2} T(t) Z_{2}=0 .
\end{aligned}
$$

Then we can rewrite equations (9) as follows:

$$
\begin{aligned}
& \left(D_{1}(t)+A_{11}(t-\tau, t) Z_{1}+A_{12}(t, t) Z_{2}\right) Z_{1}=0, \\
& \left(D_{2}(t)+A_{21}(t, t) Z_{1}+A_{22}(t, t) Z_{2}\right) Z_{2}=0
\end{aligned}
$$

where

$$
\begin{array}{ll}
D_{1}(t)=T(t) K-r_{1} T(t), & A_{11}(t-\tau, t)=a_{11} T(t-\tau) T^{*}(t), \\
A_{12}(t, t)=a_{12} T(t) T^{*}(t), & A_{22}(t, t)=a_{22} T(t) T^{*}(t), \\
D_{2}(t)=T(t) K+r_{2} T(t), & A_{21}(t, t)=-a_{21} T(t) T^{*}(t),
\end{array}
$$

and

$$
T^{*}(t)=\left[\begin{array}{cccc}
T(t) & 0 & \cdots & 0 \\
0 & T(t) & \cdots & 0 \\
\vdots & & \ddots & \vdots \\
0 & 0 & \cdots & T(t)
\end{array}\right] .
$$

By substituting the interpolation points $t_{k}$ into equations (10), we have two nonlinear systems

$$
\left(\begin{array}{c}
D_{1}\left(t_{0}\right)+A_{11}\left(t_{0}-\tau, t_{0}\right) Z_{1}+A_{12}\left(t_{0}, t_{0}\right) Z_{2} \\
D_{1}\left(t_{1}\right)+A_{11}\left(t_{1}-\tau, t_{1}\right) Z_{1}+A_{12}\left(t_{1}, t_{1}\right) Z_{2} \\
\cdot \\
\cdot \\
\cdot \\
D_{1}\left(t_{N}\right)+A_{11}\left(t_{N}-\tau, t_{N}\right) Z_{1}+A_{12}\left(t_{N}, t_{N}\right) Z_{2}
\end{array}\right) Z_{1}=0
$$

and

$$
\left(\begin{array}{c}
D_{2}\left(t_{0}\right)+A_{21}\left(t_{0}, t_{0}\right) Z_{1}+A_{22}\left(t_{0}, t_{0}\right) Z_{2} \\
D_{2}\left(t_{1}\right)+A_{21}\left(t_{1}, t_{1}\right) Z_{1}+A_{22}\left(t_{1}, t_{1}\right) Z_{2} \\
\cdot \\
\cdot \\
\cdot \\
D_{2}\left(t_{N}\right)+A_{21}\left(t_{N}, t_{N}\right) Z_{1}+A_{22}\left(t_{N}, t_{N}\right) Z_{2}
\end{array}\right) Z_{2}=0
$$


Let

$$
W_{1}=\left(\begin{array}{c}
D_{1}\left(t_{0}\right)+A_{11}\left(t_{0}-\tau, t_{0}\right) Z_{1}+A_{12}\left(t_{0}, t_{0}\right) Z_{2} \\
D_{1}\left(t_{1}\right)+A_{11}\left(t_{1}-\tau, t_{1}\right) Z_{1}+A_{12}\left(t_{1}, t_{1}\right) Z_{2} \\
\cdot \\
\cdot \\
\cdot \\
D_{1}\left(t_{N}\right)+A_{11}\left(t_{N}-\tau, t_{N}\right) Z_{1}+A_{12}\left(t_{N}, t_{N}\right) Z_{2}
\end{array}\right)
$$

and

$$
W_{2}=\left(\begin{array}{c}
D_{2}\left(t_{0}\right)+A_{21}\left(t_{0}, t_{0}\right) Z_{1}+A_{22}\left(t_{0}, t_{0}\right) Z_{2} \\
D_{2}\left(t_{1}\right)+A_{21}\left(t_{1}, t_{1}\right) Z_{1}+A_{22}\left(t_{1}, t_{1}\right) Z_{2} \\
\cdot \\
\cdot \\
\cdot \\
D_{2}\left(t_{N}\right)+A_{21}\left(t_{N}, t_{N}\right) Z_{1}+A_{22}\left(t_{N}, t_{N}\right) Z_{2}
\end{array}\right) .
$$

In addition, by the initial value, we have

$$
T\left(t_{0}\right) Z_{1}=\alpha, \quad T\left(t_{0}\right) Z_{2}=\beta .
$$

Thus, replacing first rows of the argument matrix $W_{1}, W_{2}$ by $T\left(t_{0}\right)$, we have

$$
\widetilde{W}_{1}=\left(\begin{array}{c}
T\left(t_{0}\right) \\
D_{1}\left(t_{1}\right)+A_{11}\left(t_{1}-\tau, t_{1}\right) Z_{1}+A_{12}\left(t_{1}, t_{1}\right) Z_{2} \\
\cdot \\
\cdot \\
\cdot \\
D_{1}\left(t_{N}\right)+A_{11}\left(t_{N}-\tau, t_{N}\right) Z_{1}+A_{12}\left(t_{N}, t_{N}\right) Z_{2}
\end{array}\right)
$$

and

$$
\widetilde{W}_{2}=\left(\begin{array}{c}
T\left(t_{0}\right) \\
D_{2}\left(t_{1}\right)+A_{21}\left(t_{1}-\tau, t_{1}\right) Z_{1}+A_{22}\left(t_{1}, t_{1}\right) Z_{2} \\
\cdot \\
\cdot \\
\cdot \\
D_{2}\left(t_{N}\right)+A_{21}\left(t_{N}-\tau, t_{N}\right) Z_{1}+A_{22}\left(t_{N}, t_{N}\right) Z_{2}
\end{array}\right) .
$$

Then we can rewrite equations (11) and (12) as follows:

$$
W A=B,
$$

where

$$
W=\left(\begin{array}{cc}
\widetilde{W}_{1} & 0 \\
0 & \widetilde{W}_{2}
\end{array}\right), \quad A=\left(\begin{array}{c}
Z_{1} \\
Z_{2}
\end{array}\right), \quad B=\left(\begin{array}{l}
b_{1} \\
b_{2}
\end{array}\right)
$$

and $b_{1}=[\alpha, 0,0, \ldots]^{T}, b_{2}=[\beta, 0,0, \ldots]^{T}$. 


\section{Error estimation and residual correction}

This section is devoted to checking the accuracy of our method. Since the exact solution of system (1) cannot be obtained, we will use the residual correction to obtain better approximate solutions. Residual correction is a process when the obtained approximate solution is substituted into the original equation, and a system whose solution is the error corresponding to the approximate solution is obtained. In the sequence, substituting the approximate solution $y_{s N}(t)(s=1,2)$ into system $(1)$, we obtain

$$
\begin{aligned}
& E_{1 N}(t)=y_{1 N}^{\prime}(t)-y_{1 N}(t)\left[r_{1}-a_{11} y_{1 N}(t-\tau)-a_{12} y_{2 N}(t)\right], \\
& E_{2 N}(t)=y_{2 N}^{\prime}(t)-y_{2 N}(t)\left[-r_{2}+a_{21} y_{1 N}(t)-a_{22} y_{2 N}(t)\right],
\end{aligned}
$$

where $E_{s N}(t)(s=1,2)$ denotes the residual functions.

We define the error corresponding to $y_{1 N}(t)$ and $y_{2 N}(t)$ as follows:

$$
e_{1 N}(t)=y_{1}(t)-y_{1 N}(t)
$$

and

$$
e_{2 N}(t)=y_{2}(t)-y_{2 N}(t)
$$

Substituting $y_{1}(t)$ and $y_{2}(t)$ into system (1), we have

$$
\begin{aligned}
& \left(y_{1 N}+e_{1 N}\right)^{\prime}(t)=\left(y_{1 N}+e_{1 N}\right)(t)\left[r_{1}-a_{11}\left(y_{1 N}+e_{1 N}\right)(t-\tau)-a_{12}\left(y_{2 N}+e_{2 N}\right)(t)\right], \\
& \left(y_{2 N}+e_{2 N}\right)^{\prime}(t)=\left(y_{2 N}+e_{2 N}\right)(t)\left[-r_{2}-a_{21}\left(y_{1 N}+e_{1 N}(t)-a_{22}\left(y_{2 N}+e_{2 N}\right)(t) .\right.\right.
\end{aligned}
$$

We can rewrite Eq. (18) as follows:

$$
\begin{aligned}
e_{1 N}^{\prime}(t)= & r_{1} y_{1 N}(t)-a_{11} y_{1 N}(t) e_{1 N}(t)-a_{11} y_{1 N}(t-\tau) e_{1 N}(t)-a_{11} e_{1 N}(t) e_{1 N}(t) \\
& -a_{12} y_{1 N}(t) e_{2 N}(t)-a_{21} y_{2 N}(t)-a_{12} e_{1 N}(t) e_{2 N}(t)-E_{1 N}(t), \\
e_{2 N}^{\prime}(t)= & -r_{2} y_{2 N}(t)+a_{21} y_{2 N}(t) e_{1 N}(t)+a_{21} y_{1 N} e_{2 N}(t)+a_{21} e_{2 N}(t) e_{1 N}(t) \\
& -2 a_{22} y_{2 N}(t) e_{2 N}(t)-a_{22} e_{2 N} e_{2 N}(t)-E_{2 N}(t) .
\end{aligned}
$$

Similar to system (1), this system is also nonlinear delay differential system with initial values $e_{1 N}(0)=0$ and $e_{2 N}(0)=0$. The unknown functions are $e_{1 N}(t)$ and $e_{2 N}(t)$. We will apply the method of Sect. 3 to Eq. (19) in order to obtain the approximate solutions. Let $e_{1 N, M}(t)$ and $e_{2 N, M}(t)$ be the estimation solutions of errors $e_{1 N}(t)$ and $e_{2 N}(t)$. We can obtain the new approximate solutions as follows:

$$
\begin{aligned}
& y_{1 N, M}(t)=y_{1 N}(t)+e_{1 N, M}(t), \\
& y_{2 N, M}(t)=y_{2 N}(t)+e_{2 N, M}(t) .
\end{aligned}
$$

Then $y_{1 N, M}(t)$ and $y_{2 N, M}(t)$ are the correction solutions which are more accurate than $y_{1 N}(t)$ and $y_{2 N}(t)$. We will use the residual functions to measure the accuracy of numerical 
solutions by using $y_{1 N, M}(t)$ and $y_{2 N, M}(t)$ instead of $y_{1 N}(t)$ and $y_{2 N}(t)$. In the next section we use an example to demonstrate the above idea.

\section{Numerical application}

In this section, we demonstrate our method by a detailed example. We give the values of approximate solutions $y_{s N}(t),(s=1,2)$ at selected points of the given interval for different $N$ values.

Example 1 ([44]) We consider the following system:

$$
\left\{\begin{array}{l}
y_{1}^{\prime}(t)=y_{1}(t)\left[1-y_{1}(t-\tau)-0.5 y_{2}(t)\right], \\
y_{2}^{\prime}(t)=y_{2}(t)\left[-1+2 y_{1}(t)-4 y_{2}(t)\right],
\end{array} \quad 0<t<5,\right.
$$

with $\alpha=1$ and $\beta=0.2$. In order to obtain $y_{1 N}(t)$ and $y_{2 N}(t)$ with $N=5,6$, and 7 , we apply the method of Sect. 3 for Eq. (20). Then we have

$$
\begin{aligned}
y_{15}(t)= & 0.9141-9.0596 \times 10^{-3} T_{1}^{*}(t)+9.1229 \times 10^{-2} T_{2}^{*}(t) \\
& -4.6658 \times 10^{-3} T_{3}^{*}(t)-1.6735 \times 10^{-2} T_{4}^{*}(t)+2.3208 \times 10^{-3} T_{5}^{*}(t), \\
y_{25}(t)= & 0.19536-1.1644 \times 10^{-2} T_{1}^{*}(t)+1.046 \times 10^{-2} T_{2}^{*}(t) \\
& +1.2809 \times 10^{-2} T_{3}^{*}(t)-4.6031 \times 10^{-3} T_{4}^{*}(t)+5.4663 \times 10^{-5} T_{5}^{*}(t)
\end{aligned}
$$

for $N=5$,

$$
\begin{aligned}
y_{16}(t)= & 0.91389-5.1691 \times 10^{-3} T_{1}^{*}(t)+8.3874 \times 10^{-2} T_{2}^{*}(t) \\
& -8.3620 \times 10^{-3} T_{3}^{*}(t)-1.3075 \times 10^{-2} T_{4}^{*}(t)+4.7651 \times 10^{-4} T_{5}^{*}(t) \\
& +2.2600 \times 10^{-3} T_{6}^{*}(t), \\
y_{26}(t)= & 0.195521-8.7581 \times 10^{-3} T_{1}^{*}(t)+1.0145 \times 10^{-2} T_{2}^{*}(t) \\
& +1.0997 \times 10^{-2} T_{3}^{*}(t)-3.9419 \times 10^{-3} T_{4}^{*}(t)-3.0842 \times 10^{-4} T_{5}^{*}(t) \\
& +2.0641 \times 10^{-4} T_{6}^{*}(t)
\end{aligned}
$$

for $N=6$, and

$$
\begin{aligned}
y_{17}(t)= & 0.9322-8.8302 \times 10^{-3} T_{1}^{*}(t)+8.0857 \times 10^{-2} T_{2}^{*}(t) \\
& -7.2591 \times 10^{-3} T_{3}^{*}(t)-1.1632 \times 10^{-2} T_{4}^{*}(t)-2.7616 \times 10^{-4} T_{5}^{*}(t) \\
& +1.0363 \times 10^{-3} T_{6}^{*}(t)-1.4866 \times 10^{-4} T_{7}^{*}(t), \\
y_{27}(t)= & 0.195691-9.2694 \times 10^{-3} T_{1}^{*}(t)+9.1533 \times 10^{-3} T_{2}^{*}(t) \\
& +1.0748 \times 10^{-2} T_{3}^{*}(t)-3.6569 \times 10^{-3} T_{4}^{*}(t)-1.7423 \times 10^{-4} T_{5}^{*}(t) \\
& +8.6092 \times 10^{-5} T_{6}^{*}(t)-3.3297 \times 10^{-5} T_{7}^{*}(t)
\end{aligned}
$$

for $N=7$. 


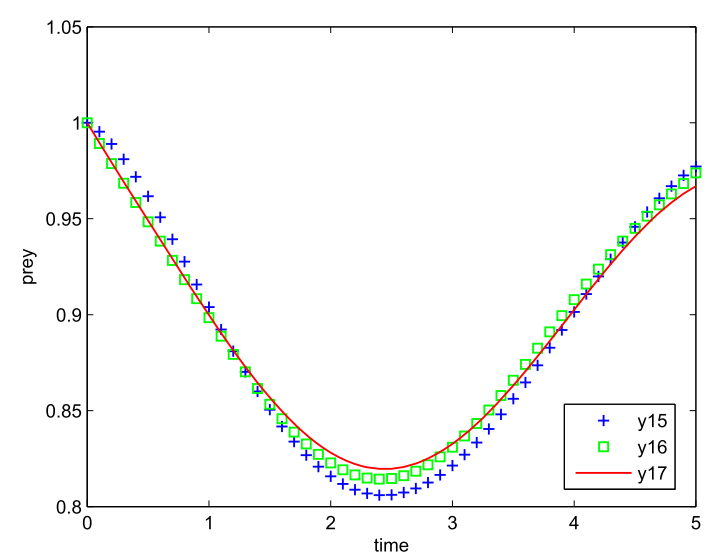

(a) By the present method.

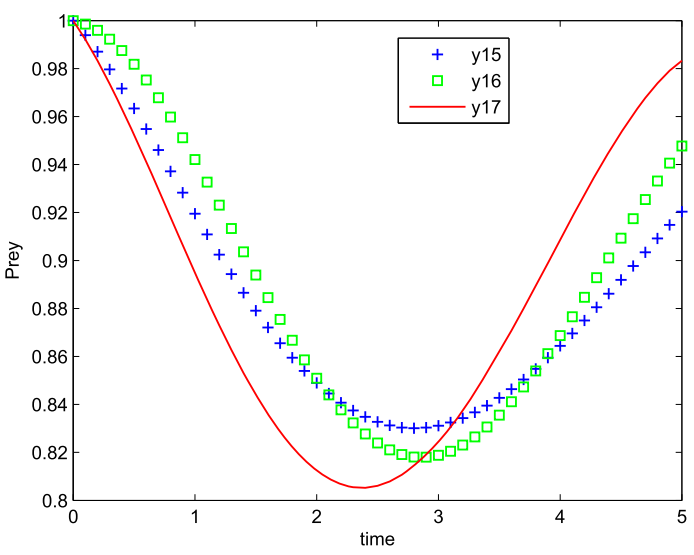

(b) By the Taylor collocation method.

Figure 1 Approximate solutions for the prey population with $N=5,6$, and 7 by the present method and the Taylor collocation method [44]

The approximate solutions of prey and predator and comparison with the results of Ref. [44] are presented in Fig. 1 and Fig. 2. Figures show that the proposed method preserves the positivity of the solutions, which is the part of the solutions of Eq. (20). To examine their accuracy, we considered the absolute residual errors of these approximate solutions. Figure 3 plots the absolute residual errors of Example 1. In Table 1, we list the absolution residual errors of the present method and Ref. [44]. It is seen from the table and figures that the absolute residual error values are decreasing as we increase the parameter $N$, which are in good agreement with the results given in Ref. [44].

For implementation residual error correction in Sect. 5, we apply again the method of Sect. 3 for Eq. (20) with choosing $N=4$ and $M=5,6$. The approximate solutions of $y_{14}(t)$ and $y_{24}(t)$ are found as follows:

$$
\begin{aligned}
y_{14}(t)= & 0.91279-1.1906 \times 10^{-2} T_{1}^{*}(t)+6.5714 \times 10^{-2} T_{2}^{*}(t) \\
& -1.4116 \times 10^{-2} T_{3}^{*}(t)-4.5221 \times 10^{-3} T_{4}^{*}(t),
\end{aligned}
$$




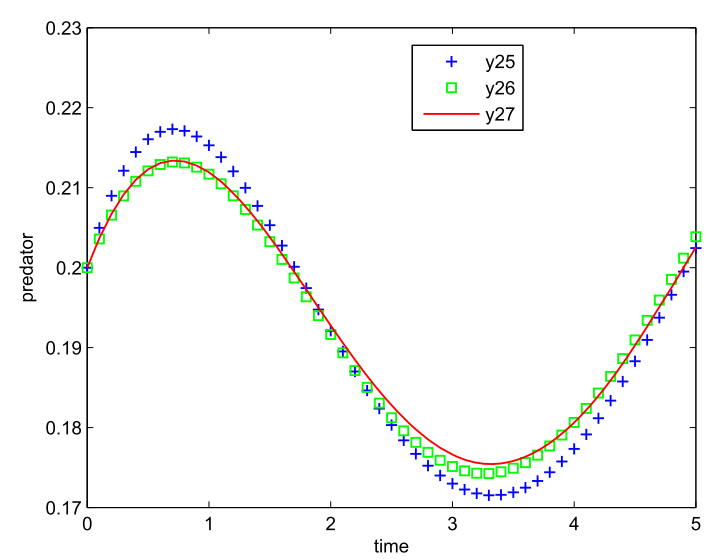

(a) By the present method.

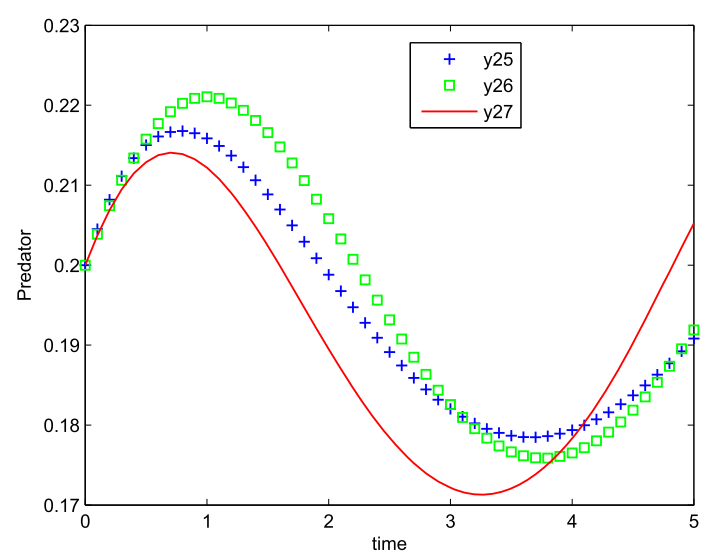

(b) By the Taylor collocation method.

Figure 2 Approximate solutions for the predator population with $N=5,6$, and 7 by the present method and the Taylor collocation method [44]

$$
\begin{aligned}
y_{24}(t)= & 0.19698-6.3365 \times 10^{-3} T_{1}^{*}(t)+7.2034 \times 10^{-3} T_{2}^{*}(t) \\
& +7.5775 \times 10^{-3} T_{3}^{*}(t)-2.9467 \times 10^{-3} T_{4}^{*}(t) .
\end{aligned}
$$

To realize the error approximate concept in Sect. 5 with $N=4$ and $M=5,6$, the estimated errors are obtained, namely

$$
\begin{aligned}
e_{14,5}(t)= & 0.0013143+2.8461 \times 10^{-3} T_{1}^{*}(t)+2.5515 \times 10^{-2} T_{2}^{*}(t) \\
& -9.4498 \times 10^{-3} T_{3}^{*}(t)-1.2212 \times 10^{-2} T_{4}^{*}(t)+2.3208 \times 10^{-3} T_{5}^{*}(t), \\
e_{24,5}(t)= & -0.0016213-5.3077 \times 10^{-3} T_{1}^{*}(t)+3.2564 \times 10^{-3} T_{2}^{*}(t) \\
& +5.2317 \times 10^{-3} T_{3}^{*}(t)-1.6564 \times 10^{-3} T_{4}^{*}(t)+5.4663 \times 10^{-5} T_{5}^{*}(t), \\
e_{14,6}(t)= & 2.8176 \times 10^{-3}+5.1572 \times 10^{-2} T_{1}^{*}(t)+1.557 \times 10^{-2} T_{2}^{*}(t) \\
& -3.149 \times 10^{-2} T_{3}^{*}(t)+1.6031 \times 10^{-4} T_{4}^{*}(t)-2.6291 \times 10^{-4} T_{5}^{*}(t)
\end{aligned}
$$




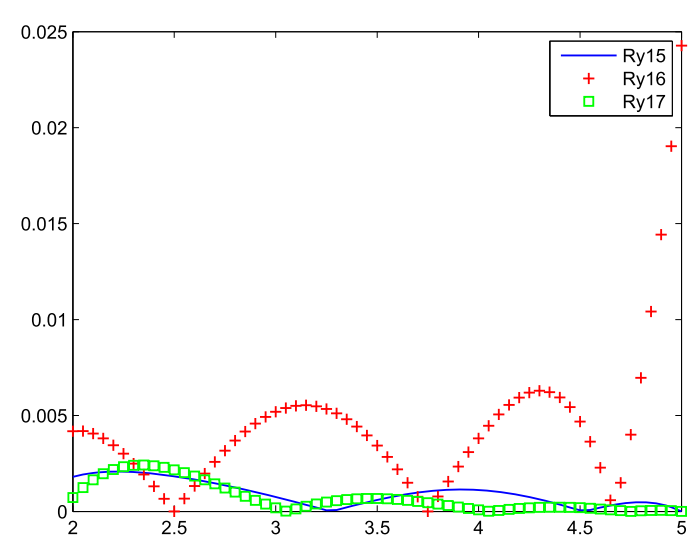

(a) Residual error of prey population.

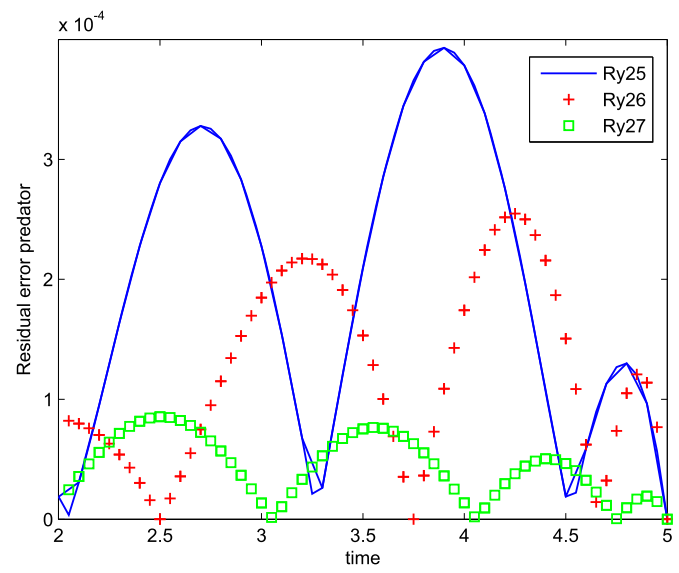

(b) Residual error of predator population.

Figure 3 Absolute residual errors corresponding to the approximate solutions of prey and predator population with $N=5,6$, and 7

$$
\begin{aligned}
+ & 1.2712 \times 10^{-3} T_{6}^{*}(t), \\
e_{24,6}(t)= & -1.0873 \times 10^{-3}+1.0813 \times 10^{-2} T_{1}^{*}(t)+1.3781 \times 10^{-2} T_{2}^{*}(t) \\
& -2.2536 \times 10^{-3} T_{3}^{*}(t)-3.2036 \times 10^{-3} T_{4}^{*}(t)+7.4238 \times 10^{-4} T_{5}^{*}(t) \\
& -1.8855 \times 10^{-4} T_{6}^{*}(t) .
\end{aligned}
$$

Then we can obtain our improved approximate solutions:

$$
y_{14,5}(t)=y_{14}(t)+e_{14,5}(t), \quad y_{24,5}(t)=y_{24}(t)+e_{24,5}(t)
$$

and

$$
y_{14,6}(t)=y_{14}(t)+e_{14,6}(t), \quad y_{24,6}(t)=y_{24}(t)+e_{24,6}(t) .
$$


Table 1 Comparison of absolute errors obtained by the present method and [44] for Example 1

\begin{tabular}{|c|c|c|c|c|c|c|}
\hline \multirow[t]{3}{*}{$t$} & \multicolumn{6}{|c|}{ Residual errors $e_{1 N}$} \\
\hline & \multicolumn{3}{|c|}{ Present method } & \multicolumn{3}{|l|}{ Ref. [44] } \\
\hline & $N=5$ & $N=6$ & $N=7$ & $N=5$ & $N=6$ & $N=7$ \\
\hline 0 & $3.4405 e-01$ & $4.9083 \mathrm{e}-01$ & $3.7236 \mathrm{e}-01$ & $4.294 \mathrm{e}-02$ & $5.503 e-03$ & $2.670 \mathrm{e}-03$ \\
\hline 1 & $3.3903 \mathrm{e}-02$ & $5.8991 \mathrm{e}-02$ & $5.4357 \mathrm{e}-02$ & $7.429 \mathrm{e}-03$ & $1.227 \mathrm{e}-03$ & $8.449 e-04$ \\
\hline 2 & $1.7890 \mathrm{e}-03$ & $4.1996 \mathrm{e}-03$ & $7.2944 \mathrm{e}-04$ & $1.424 \mathrm{e}-02$ & $8.640 e-04$ & $6.861 e-04$ \\
\hline 3 & $7.2261 \mathrm{e}-04$ & $5.3871 \mathrm{e}-03$ & $1.9676 \mathrm{e}-04$ & $1.533 \mathrm{e}-02$ & $1.796 \mathrm{e}-04$ & $6.649 \mathrm{e}-04$ \\
\hline 4 & $1.0870 \mathrm{e}-03$ & $4.0386 \mathrm{e}-03$ & $7.9089 e-05$ & $9.235 \mathrm{e}-03$ & $1.864 \mathrm{e}-04$ & $6.439 e-04$ \\
\hline 5 & $1.2145 \mathrm{e}-04$ & $2.4864 \mathrm{e}-02$ & $2.8842 \mathrm{e}-06$ & $3.087 \mathrm{e}-02$ & $2.740 \mathrm{e}-03$ & $1.698 e-03$ \\
\hline
\end{tabular}

\begin{tabular}{|c|c|c|c|c|c|c|}
\hline \multirow[t]{3}{*}{ t } & \multicolumn{6}{|c|}{ Residual errors $e_{2 N}$} \\
\hline & \multicolumn{3}{|c|}{ Present method } & \multicolumn{3}{|l|}{ Ref. [44] } \\
\hline & $N=5$ & $N=6$ & $N=7$ & $N=5$ & $N=6$ & $N=7$ \\
\hline 0 & $1.4697 \mathrm{e}-02$ & $1.0400 \mathrm{e}-03$ & $1.7618 \mathrm{e}-04$ & $4.405 e-03$ & $1.422 \mathrm{e}-04$ & $1.929 \mathrm{e}-04$ \\
\hline 1 & $1.4982 \mathrm{e}-03$ & $6.1149 \mathrm{e}-05$ & $1.2629 \mathrm{e}-06$ & $1.331 \mathrm{e}-03$ & $3.395 e-05$ & $3.404 \mathrm{e}-05$ \\
\hline 2 & $3.7072 \mathrm{e}-05$ & $6.4177 \mathrm{e}-05$ & $3.4964 \mathrm{e}-05$ & $1.164 \mathrm{e}-03$ & $7.251 \mathrm{e}-05$ & $1.155 \mathrm{e}-04$ \\
\hline 3 & $2.3864 e-04$ & $1.3460 \mathrm{e}-04$ & $1.4228 \mathrm{e}-05$ & $3.859 \mathrm{e}-04$ & $1.711 \mathrm{e}-04$ & $1.751 \mathrm{e}-04$ \\
\hline 4 & $3.0443 e-04$ & $3.6073 e-04$ & $3.0842 \mathrm{e}-05$ & $1.442 \mathrm{e}-04$ & $2.116 \mathrm{e}-04$ & $1.587 \mathrm{e}-04$ \\
\hline 5 & $1.4743 e-04$ & $1.3612 \mathrm{e}-04$ & $4.0033 e-06$ & $4.583 e-03$ & $9.451 \mathrm{e}-04$ & $7.441 \mathrm{e}-06$ \\
\hline
\end{tabular}

Table 2 Absolute residual error values of $y_{s 4, M}(t)$ with $s=1,2$ and $N=4$ and $M=5,6$ at some points (for comparison, one can see [44])

\begin{tabular}{|c|c|c|c|c|c|c|}
\hline \multirow[t]{2}{*}{$t$} & \multicolumn{3}{|l|}{$e_{14, M}$} & \multicolumn{3}{|l|}{$e_{24, M}$} \\
\hline & $N=4$ & $N=4, M=5$ & $N=4, M=6$ & $N=4$ & $N=4, M=5$ & $N=4, M=6$ \\
\hline 0 & $1.8532 \mathrm{e}-01$ & $3.4677 \mathrm{e}-01$ & 7.6478e-01 & 7.9220e-03 & $1.4726 \mathrm{e}-02$ & $3.1173 \mathrm{e}-03$ \\
\hline 0.5 & $1.2514 \mathrm{e}-01$ & $1.2920 \mathrm{e}-01$ & $3.4068 \mathrm{e}-01$ & $2.1792 \mathrm{e}-03$ & $2.6024 \mathrm{e}-04$ & $9.5723 \mathrm{e}-04$ \\
\hline 1 & $7.5154 \mathrm{e}-02$ & $3.4368 \mathrm{e}-02$ & $1.3965 \mathrm{e}-01$ & $1.6174 \mathrm{e}-03$ & $1.5337 \mathrm{e}-03$ & $8.3699 \mathrm{e}-04$ \\
\hline 1.5 & $3.8252 \mathrm{e}-02$ & $4.1932 \mathrm{e}-03$ & $4.8231 \mathrm{e}-02$ & $2.2729 \mathrm{e}-03$ & $2.7637 \mathrm{e}-04$ & $7.1159 \mathrm{e}-04$ \\
\hline 2 & $1.3902 \mathrm{e}-02$ & $1.8065 \mathrm{e}-03$ & $1.1406 \mathrm{e}-02$ & $1.1969 \mathrm{e}-03$ & $1.8752 \mathrm{e}-05$ & $9.6725 \mathrm{e}-04$ \\
\hline 2.5 & $8.0535 \mathrm{e}-11$ & $1.8300 \mathrm{e}-03$ & $1.5884 \mathrm{e}-10$ & $2.6391 \mathrm{e}-11$ & $2.8032 \mathrm{e}-04$ & $7.7868 \mathrm{e}-09$ \\
\hline 3 & $5.8527 \mathrm{e}-03$ & $7.5528 \mathrm{e}-04$ & $1.5773 \mathrm{e}-03$ & $5.0326 \mathrm{e}-04$ & $2.2769 \mathrm{e}-04$ & $7.8870 \mathrm{e}-04$ \\
\hline 3.5 & $5.8543 \mathrm{e}-03$ & $5.9818 \mathrm{e}-04$ & $5.6770 \mathrm{e}-04$ & $3.6513 \mathrm{e}-04$ & $2.0922 \mathrm{e}-04$ & $4.5973 \mathrm{e}-04$ \\
\hline 4 & $2.2951 \mathrm{e}-03$ & $1.1248 \mathrm{e}-03$ & $3.9443 e-04$ & 7.3925e-05 & $3.7841 \mathrm{e}-04$ & $3.9797 e-04$ \\
\hline 4.5 & $1.5116 \mathrm{e}-03$ & $6.3471 \mathrm{e}-05$ & $2.6966 \mathrm{e}-04$ & $5.3039 \mathrm{e}-06$ & $1.8942 \mathrm{e}-05$ & $2.6853 \mathrm{e}-04$ \\
\hline 5 & $8.6549 \mathrm{e}-11$ & $2.1856 \mathrm{e}-10$ & $1.3063 e-09$ & $2.3356 \mathrm{e}-11$ & $4.1398 \mathrm{e}-10$ & $2.0789 \mathrm{e}-04$ \\
\hline
\end{tabular}

The improvement values of $y_{14}(t)$ and $y_{24}(t)$ with $M=5,6$ are given in Fig. 4 . It revealed that the proposed technique preserved the positive solutions of the given delayed prey-predator system. In order to understand how much improvement is provided by this scheme, the absolute residual errors of the original approximate solutions $y_{14}(t)$ and $y_{24}(t)$ are shown together with those of corrected solutions in Fig. 5. In Table 2, we list the residual errors of the improvement solutions at some point on our interval. In view of Fig. 5 and Table 2, absolute residual errors of $y_{14,5}(t)$ and $y_{24,5}(t)$ are smaller than those of $y_{14}(t)$ and $y_{24}(t)$ respectively, and absolute residual errors of $y_{14,6}(t)$ and $y_{24,6}(t)$ are smaller. Hence, we can comment that residual correction in general provides a certain improvement in the approximate solutions for Eq. (20). 


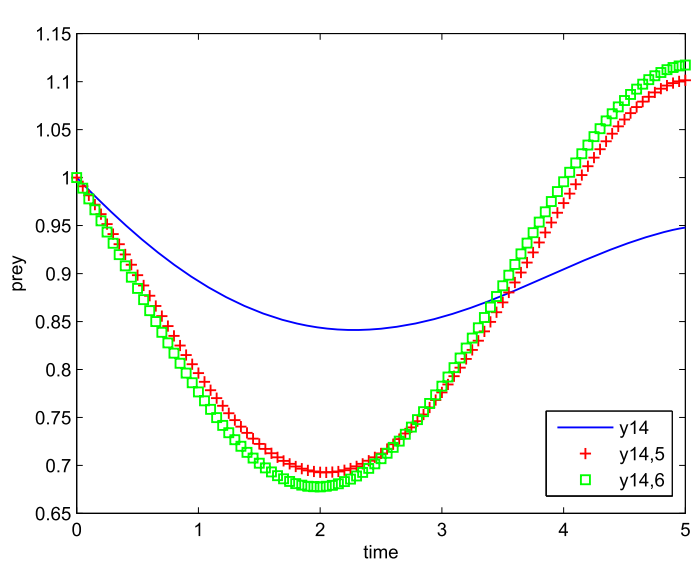

(a) Approximate solutions of prey.

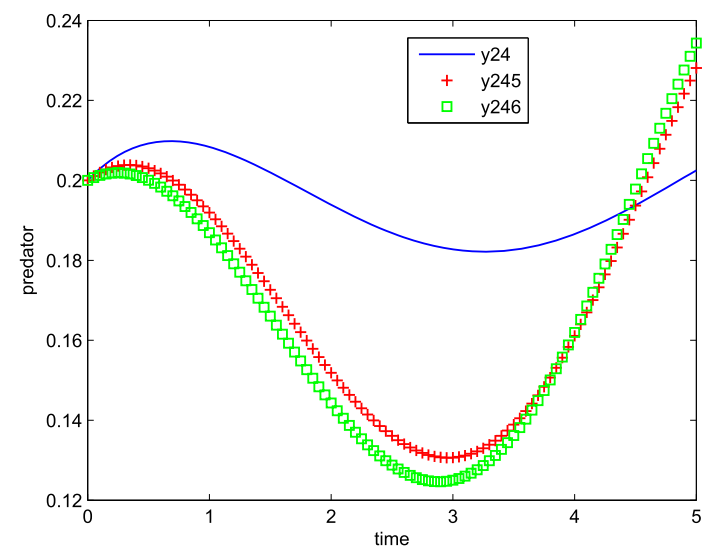

(b) Approximate solutions of predator.

Figure 4 Graphics of the approximate solutions for the prey and predator with $N=4$ and their two improvements obtained by $M=5,6$

\section{Conclusion}

In this paper, a modified Chebyshev collocation method based on the residual correction technique is presented to solve the Lotka-Volterra model with delay. An efficient error estimation can be made by using this technique. The key advantages of this approach are its low-cost computing and simplicity of implementation. Also the present method has the ability to convert the given problem into a system of mathematical equations, which can be solved easily using MATLAB or MAPLE software. Our numerical results are compared with the numerical results of [44]. The results show that they are in good correspondence with the results obtained in [44]. Based on the above facts, the modified Chebyshev collocation method is a powerful mathematical tool to obtain the numerical solutions of a nonlinear system. In future the proposed method will be applied to the fractional Lotka-Volterra biological model with and without a time delay. It is hoped that the biological relevance of the numerical results, such as stability and chaotic behavior, can be obtained. Similarly, numerical techniques may be designed for fractional reaction diffusion systems. 


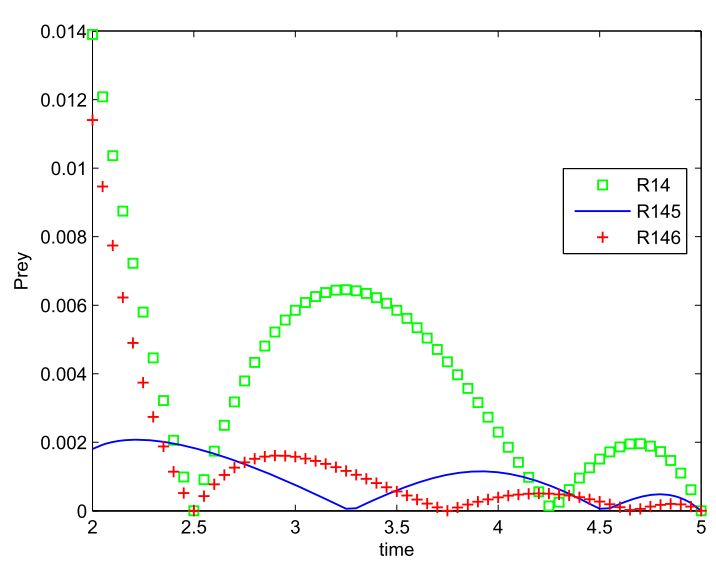

(a) Absolute residual errors of prey.

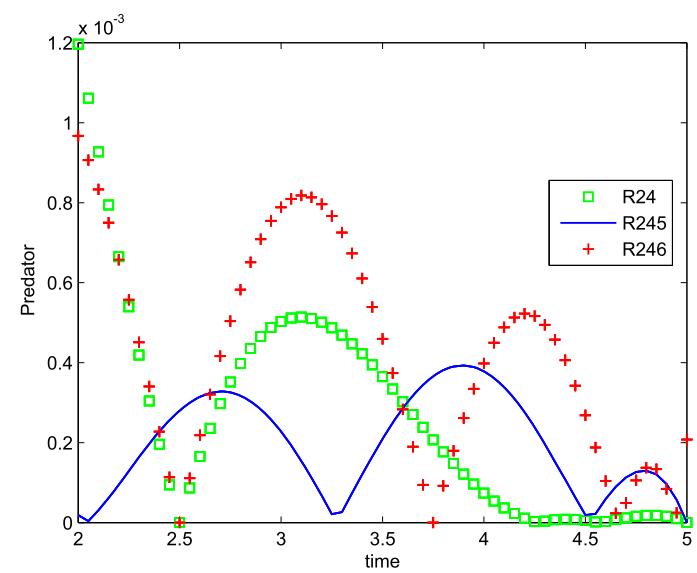

(b) Absolute residual errors of predator.

Figure 5 Absolute residual errors for the prey and predator population corresponding to the approximate solutions with $N=4$ and their improvement $M=5,6$

\section{Acknowledgements}

The authors would like to thank the editor and the anonymous referees for their helpful comments.

Funding

This work is supported by the Fundamental Research Funds for Central Universities (2572018BC19).

Availability of data and materials

Not applicable.

Competing interests

The authors declare that they have no competing interests.

Authors' contributions

All authors equally contributed in making the first draft and revision and they read and approved the final manuscript.

\section{Publisher's Note}

Springer Nature remains neutral with regard to jurisdictional claims in published maps and institutional affiliations.

Received: 17 April 2020 Accepted: 11 June 2020 Published online: 24 June 2020

\section{References}

1. Lotka, A.J.: Elements of Physical Biology. Williams \& Wilkins, Baltimore (1925) 
2. Volterra, $V_{\text {:: }}$ Fluctuations in the abundance of a species considered mathematically. Nature 118, 558-560 (1926)

3. Craciun, G., Nazarov, F., Pantea, C.: Persistence and permanence of mass-action and power-law dynamical systems. SIAM J. Appl. Math. 73, 305-329 (2013)

4. Xie, L., Wang, Y.: On a fully parabolic chemotaxis system with Lotka-Volterra competitive kinetics. J. Math. Anal. Appl. 471, 584-598 (2019)

5. Su, K., Wang, C., Zhang, S., Liu, S.: Lotka-Volterra equation based modeling of aerobic granulation process in sequencing batch reactors. Int. Biodeterior. Biodegrad. 115, 49-54 (2016)

6. Cropp, R.A., Norbury, J.: Population interactions in ecology: a rule-based approach to modeling ecosystems in a mass-conserving framework. SIAM Rev. 57, 437-446 (2015)

7. Li, C., Zhu, H.: Canard cycles for predator-prey systems with Holling types of functional response. J. Differ. Equ. 254, 879-910 (2013)

8. Zhu, C., Yin, G.: On competitive Lotka-Volterra model in random environments. J. Math. Anal. Appl. 357, 154-170 (2009)

9. Badri, V., Yazdanpanah, M.J., Tavazoei, M.S.: Global stabilization of Lotka-Volterra systems with interval uncertainty. IEEE Trans. Autom. Control 64, 1209-1213 (2018)

10. May, R.M.: Time-delay versus stability in population models with two and three trophic levels. Ecology 54, 315-325 (1973)

11. Yan, X.P., Chu, Y.D.: Stability and bifurcation analysis for a delayed Lotka-Volterra predator-prey system. J. Comput. Appl. Math. 196, 198-210 (2006)

12. Ruan, S.: On nonlinear dynamics of predator-prey models with discrete delay. Math. Model. Nat. Phenom. 4, 140-188 (2009)

13. Capobianco, G., Conte, D., Del Prete, I.: High performance parallel numerical methods for Volterra equations with weakly singular kernels. J. Comput. Appl. Math. 228, 571-579 (2013)

14. Paul, S., Mondal, S.P., Bhattacharya, P.: Numerical solution of Lotka Volterra prey predator model by using Runge-Kutta-Fehlberg method and Laplace Adomian decomposition method. Alex. Eng. J. 55, 613-617 (2016)

15. Gokmen, E., Isik, O.R., Sezer, M.: Taylor collocation approach for delayed Lotka-Volterra predator-prey system. Appl. Math. Comput. 268, 671-684 (2015)

16. Shahmorad, S., Ostadzad, M.H., Baleanu, D.: A tau-like numerical method for solving fractional delay integro-differential equations. Appl. Numer. Math. 151, 322-336 (2020)

17. Singh, J., Kumar, D., Baleanu, D.: A new analysis of fractional fish farm model associated with Mittag-Leffler-type kernel. Int. J. Biomath. 13(2), 2050010 (2020)

18. Abdel-Gawad, H.I., Tantawy, M., Baleanu, D.: Fractional KdV and Boussenisq-Burger's equations, reduction to PDE and stability approaches. Math. Methods Appl. Sci. 43, 4125-4135 (2020)

19. Jawaz, M., Ahmed, N., Baleanu, D., Rafiq, M., Rehman, M.A.: Positivity preserving technique for the solution of HIV/AIDS reaction diffusion model with time delay. Front. Phys. 7, 229 (2020)

20. Farooq, U., Khan, H., Baleanu, D., Arif, M.: Numerical solutions of fractional delay differential equations using Chebyshev wavelet method. Comput. Appl. Math. 38, 195 (2019). https://doi.org/10.1007/s40314-019-0953-y

21. Emile, F., Goufo, D., Kumar, S., Mugisha, S.B.: Similarities in a fifth-order evolution equation with and with no singular kernel. Chaos Solitons Fractals 130, 109467 (2020)

22. Ghanbari, B., Kumar, S., Kumar, R.: A study of behaviour for immune and tumor cells in immunogenetic tumour model with non-singular fractional derivative. Chaos Solitons Fractals 133, 109619 (2020)

23. Veeresha, P., Prakasha, G., Kumar, S.: A fractional model for propagation of classical optical solitons by using nonsingular derivative. Math. Methods Appl. Sci. 1-15 (2020, in press)

24. Kumar, S., Ghosh, S., Samet, B., Goufo, E.F.D.: An analysis for heat equations arises in diffusion process using new Yang-Abdel-Aty-Cattani fractional operator. Math. Methods Appl. Sci. 43(9), 6062-6080 (2020)

25. Kumar, S., Ahmadian, A., Kumar, R., Kumar, D., Singh, J., Baleanu, D., Salimi, M.: An efficient numerical method for fractional SIR epidemic model of infectious disease by using Bernstein wavelets. J. Math. 8, 558 (2020). https://doi.org/10.3390/math8040558

26. Alshabanat, A., Jleli, M., Kumar, S., Samet, B.: Generalization of Caputo-Fabrizio fractional derivative and applications to electrical circuits. Front. Phys. 8, 64 (2020). https://doi.org/10.3389/fphy.2020.00064

27. Mustahsan, M., Kiran, A., Sing, J., Nisar, K.S., Kumar, D.: Higher order B-spline differential quadrature rule to approximate generalized Rosenau-RLW equation. Math. Methods Appl. Sci. 43(11), 6812-6822 (2020)

28. Kumar, S., Kumar, R., Singh, J., Nisar, K.S., Kumar, D.: An efficient numerical scheme for fractional model of HIV-1 infection of CD4+ T-cells with the effect of antiviral drug therapy. Alex. Eng. J. (2020, in press). https://doi.org/10.1016/j.aej.2019.12.046

29. Bhatter, S., Mathur, A., Kumar, D., Nisar, K.S., Singh, J.: Fractional modified Kawahara equation with Mittag-Leffler law. Chaos Solitons Fractals 131, 109508 (2019). https://doi.org/10.1016/j.chaos.2019.109508

30. Dubey, V.P., Kumar, R., Kumar, D., Khan, I., Singh, J.: An efficient computational scheme for nonlinear time fractional systems of partial differential equations arising in physical sciences. Adv. Differ. Equ. 2020, 46 (2020)

31. Kumar, S., Kumar, A., Odibat, Z.M.: A nonlinear fractional model to describe the population dynamics of two interacting species. Math. Methods Appl. Sci. 40, 4134-4148 (2017)

32. Kumar, S., Kumar, R., Agarwal, R.P., Samet, B.: A study of fractional Lotka-Volterra population model using Haar wavelet and Adams-Bashforth-Moulton methods. Math. Methods Appl. Sci. 43, 5564-5578 (2020)

33. Kumar, S., Kumar, R., Cattani, C., Samet, B.: Chaotic behaviour of fractional predator-prey dynamical system. Chaos Solitons Fractals 135, 109811 (2020)

34. Singh, J., Kilicman, A., Kumar, D., Swroop, R.: Numerical study for fractional model of nonlinear predator-prey biological population dynamic system. Therm. Sci. 23, 2017-2025 (2019)

35. Ghimire, B.K., Tian, H.Y., Lamichhane, A.R.: Numerical solutions of elliptic partial differential equations using Chebyshev polynomials. Comput. Math. Appl. 72, 1042-1054 (2016)

36. Li, Z., Sun, G., He, C., Liu, X., Zhang, R., Li, Y., Zhao, D., Liu, H., Zhang, F.: Multi-variable regression methods using modified Chebyshev polynomials of class 2. J. Comput. Appl. Math. 346, 609-619 (2019)

37. Djukić, D.L., Reichel, L., Spalević, M.M.: Internality of generalized averaged Gaussian quadrature rules and truncated variants for measures induced by Chebyshev polynomials. Appl. Numer. Math. 142, 190-205 (2019) 
38. Fox, L., Parker, I.B.: Chebyshev Polynomials in Numerical Analysis. Oxford University Press, London (1968)

39. Boyd, J.P.: Chebyshev and Fourier Spectral Methods. Dover, New York (2000)

40. Mason, J.C., Handscomb, D.C.: Chebyshev Polynomials. Chapman \& Hall/CRC, Florida (2003)

41. Handscomb, D.C.: Chebyshev Polynomials. Chapman \& Hall/CRC, 2, 9 (2003)

42. Synder, M.A.: Chebyshev methods. In: Numerical Approximation, Prentice Hall, Englewood Cliffs, pp. $106-109$ (1966)

43. Yang, C.: Modified Chebyshev collocation method for pantograph-type differential equations. Appl. Numer. Math. 134, 132-144 (2018)

44. Yüzbaş, Ş., Karaçayır, M.: A numerical method for solutions of Lotka-Volterra predator-prey model with time-delay. Int. J. Biomath. 11, 1-16 (2018)

Submit your manuscript to a SpringerOpen ${ }^{\odot}$ journal and benefit from:

- Convenient online submission

Rigorous peer review

- Open access: articles freely available online

- High visibility within the field

- Retaining the copyright to your article

Submit your next manuscript at $\gg$ springeropen.com 